\title{
Minor components modulate sensitivity to the pheromone antagonist Z11-14:Ac in male lightbrown apple moth, Epiphyas postvittana (Lepidoptera: Tortricidae) in the field
}

\author{
Rachael M. Horner ${ }^{1, \star}$, Thomas E.S. Sullivan ${ }^{1}$, Andrew M. Sporle ${ }^{1}$, Lloyd D. Stringer ${ }^{1,2}$, \\ Lee-Anne M. Manning ${ }^{1}$, Ashraf M. El-Sayed ${ }^{1}$ and David Maxwell Suckling ${ }^{1,2}$ \\ ${ }^{1}$ The New Zealand Institute for Plant \& Food Research Limited, Private Bag 4704, Christchurch, \\ New Zealand \\ ${ }^{2}$ School of Biological Sciences, University of Auckland, Tāmaki Campus, Building 733, \\ Auckland, New Zealand \\ ${ }^{*}$ Corresponding author: rachael.horner@plantandfood.co.nz
}

\begin{abstract}
Epiphyas postvittana is a major horticultural pest in many countries, including New Zealand. Recently, two minor components (E)-11-tetradecen-1-ol and (E)-11-hexadecenyl acetate were found to increase the attraction of a previously identified two-component sex pheromone (95:5 blend of (E)-11-tetradecenyl acetate [E11-14:Ac] and (E,E)-9,11tetradecadienyl acetate [E9,E11-14:Ac]) of Epiphyas postvittana. We hypothesised that the interaction between these minor components and the behavioural antagonist $(Z)$ 11-tetradecenyl acetate (Z11-14:Ac) would modulate its antagonistic effect. The effect of increasing the ratio (0.5-10\%) of Z11-14:Ac was tested in traps baited with E11-14:Ac and E9,E11-14:Ac (95:5), with or without the addition of the two minor compounds. Catch decreased as the percentage of Z11-14:Ac increased ( $\chi^{2}=108.74$; d.f. $=9$; $\left.P<0.001\right)$. Overall, more moths were caught in traps baited with four- vs two-component lures $\left(\chi^{2}=9.81\right.$; d.f. $=1$; $\mathrm{P}=0.002)$; in pair-level comparisons, significant differences in catch number between the two- and four-component lures were observed when the E11-14:Ac: Z11-14:Ac ratio was 99:1 $(\mathrm{P}=0.031)$ or $98: 2(\mathrm{P}=0.047)$. The addition of the two minor components mitigated the reduced catch effect due to lower proportions of E11-14:Ac and the presence of 1-2\% antagonist.
\end{abstract}

Keywords Epiphyas postvittana, lightbrown apple moth, Lepidoptera, Tortricidae, pheromone, inhibitor, mating disruption.

\section{INTRODUCTION}

The Australian species, Epiphyas postvittana, the lightbrown apple moth (Lepidoptera: Tortricidae) is a major pest in many countries, including New Zealand, Australia and the USA. It feeds on many different plants, including fruit crops, vegetables and ornamentals, with the larvae causing significant damage to leaves and fruits. Each application of leafroller-specific pesticides costs growers around NZ\$100 per hectare, and the use of mating disruption with sex pheromones can result in a reduction of up to two spray applications each year. Many insects use multicomponent sex pheromones to discriminate among potential mating partners and, in moths, the blend is normally dominated by one or two major components. However, the inclusion of further minor components can optimise the behavioural response to the pheromone.

The effects of pheromone antagonists on 
behaviour vary among species, presumably because of different evolutionary factors during sexual selection within a genus (Martin et al. 2016). For example, in Helicoverpa zea, olfactory receptor neurons detect both its sex pheromones and the sex pheromones (antagonists) that are used by closely related species (Cossé et al. 1998). This prevents insects accidentally mating with a closely related species. Field trapping of a related species complex also showed the antagonistic effect of heterospecific sex pheromone compounds by reducing catch of the target species (Juárez et al. 2016). Sometimes antagonists are chemical analogues with the same carbon chain length and double bond configuration but a different functional group (Renou \& Guerrero 2000). Antagonism can also result from a difference in the ratio of components (Wu et al. 2015). Behavioural responses can vary unpredictably, for example, Cydia pomonella is highly responsive to the antagonist codlemone acetate but a sister species (Cydia succedana) that uses codlemone acetate as its sex pheromone is not antagonised by the codlemone (Stephens et al. 2008).

The pheromone of Epiphyas postvittana, was originally incompletely identified as a twocomponent blend of 95\% (E)-11-tetradecenyl acetate (E11-14:Ac) and 5\% (E,E)-9,11tetradecadienyl acetate (E9,E11-14:Ac) by Bellas et al. (1983). Subsequently, a blend of these two compounds plus (E)-11-tetradecen-1-ol and (E)-11-hexadecenyl acetate was reported to have superior attraction (El-Sayed et al. 2011). Another compound, (Z)-11-tetradecenyl acetate (Z1114:Ac), an isomer of E11-14:Ac, has been shown to inhibit male attraction to pheromone traps. The effectiveness of the E11-14:Ac/E9,E11-14:Ac blend in attracting male $E$. postvittana to traps was found to be reduced when 10\% of Z11-14:Ac was included in the pheromone loading (Rumbo et al. 1993; Stephens et al. 2008). The Z11-14:Ac isomer is not present in the sex pheromone gland of E. postvittana, but is a pheromone component of many related Australian species (Horak et al. 1988), and could mediate species isolation (Rumbo et al. 1993).
The two-component (95:5 E11-14:Ac and E9,E11-14:Ac) blend has been used successfully in behavioural disruption of E. postvittana (Suckling et al. 1990; Mo et al. 2006). However, the early commercially available mating disruption dispensers developed in New Zealand (Suckling \& Shaw 1995) contained approximately $20 \%$ of the Z11-14:Ac isomer, which was produced as a by-product of the synthesis of the E-isomer and not removed prior to use. The presence of significant amounts of Z11-14:Ac may reduce the level of behavioural disruption achieved, due to the loss of competitive attraction as a mechanism for behavioural disruption (Suckling et al. 2012).

Wind tunnel studies have shown that inhibitory effects on attraction could be reduced if a pheromone and an antagonist were not released from the same physical source (Rumbo et al. 1993). Therefore, the aim of this study was to determine the sensitivity of E. postvittana to antagonism by Z11-14:Ac during attraction in the field, using either the two- or four-component sex pheromone blends detailed above, in order to determine how the attractive effect of minor components was affected by the antagonist.

\section{MATERIALS AND METHODS}

\section{Lures}

All compounds were purchased from the Pherobank (Wageningen, The Netherlands). The isomeric purities of the individual compounds were: E11-14:Ac 99.5\%; E9,E11 14:Ac 98.8\%; (E)11-tetradecen-1-ol 95.8\%; (E)-11-hexadecenyl acetate 99.9\%; and Z11-14:Ac 99.5\%. Purities were determined using a Saturn 2200 gas chromatography/mass spectrometry GC/MS (Varian Inc., Walnut Creek, CA, USA) equipped with a $30 \mathrm{~m} \times 0.25 \mathrm{~mm}$ internal diameter DB$5 \mathrm{~ms}$ capillary column (Varian Inc.). Helium was used as the carrier gas and was forced through the column at a rate of $1 \mathrm{~mL} / \mathrm{min}$. Samples were injected in splitless mode, and the temperature of the injection port was maintained at $220{ }^{\circ} \mathrm{C}$. The oven was programmed to ramp the temperature from $80^{\circ} \mathrm{C}$ ( 1 min hold) to $220^{\circ} \mathrm{C}$ ( 2 min hold $)$ at a rate of $10^{\circ} \mathrm{C} / \mathrm{min}$, then from $220^{\circ} \mathrm{C}$ to $280^{\circ} \mathrm{C}$ (10 min hold) at a rate of $20^{\circ} \mathrm{C} / \mathrm{min}$. Mass spectra 
were recorded at an ionisation voltage of $70 \mathrm{eV}$ over a mass range of 20 to $499 \mathrm{~m} / z$. The transfer line and the ion trap were held at $280{ }^{\circ} \mathrm{C}$ and 180 ${ }^{\circ} \mathrm{C}$ respectively. Two-component lures contained 95:5 E11-14:Ac: E9,E11 14:Ac. Four-component lures were composed of E11-14:Ac and E9,E11 14:Ac as above but also contained (E)-11tetradecen-1-ol and (E)-11-hexadecenyl acetate at a ratio of 95:5:1:0.5 (El-Sayed et al. 2011). In each case, $1 \mathrm{mg}$ of the blend in hexane was loaded onto rubber septa (West Pharmaceutical Services, Kearney, NE, USA). Both lure types also received Z11-14:Ac at levels of 0.5, 0.7, 0.85, 1.0, 1.5, 2.0, 3.0, 4.0, 5.0 and $10.0 \%$ of the E11-14:Ac load (Fig. 1). Original purities of compounds were taken into account when calculating amounts to be added to each treatment.

\section{Field experiment}

The field experiment was conducted in a vineyard in Waipara, North Canterbury $\left(43^{\circ}\right.$ $\left.3.3^{\prime} \mathrm{S}, 172^{\circ} 45.6^{\prime} \mathrm{E}\right)$ that had a high population of E. postvittana. This experiment compared catches of male E. postvittana in traps baited with the different lures. Blank traps without lures were used as negative controls. There were seven traps (replicates) per treatment arranged $20 \mathrm{~m}$ apart in a randomised block design. The traps were operated from 21 February to 15 March 2017. Traps were serviced weekly, with insects counted and lures and bases changed.

\section{Statistical analyses.}

The effect of a change in the E11-14:Ac : Z11-14:Ac ratio used and the presence of the additional compounds on the catch of male $E$. postvittana was investigated. As the data did not conform to assumptions of normality, a Generalised Linear Model (GLM) with a Poisson distribution logarithm-link function was used to determine differences among treatments. Pairwise comparisons between 2- and 4-component catches for each E11-14:Ac: Z11-14:Ac ratio were made using Wilcoxon matched-pairs tests. Un-baited control traps were removed from analyses as no moths were caught in them and because the focus of this study was on the role of E11-14:Ac : Z11-14:Ac ratio and the effect of minor components on catch. All analyses were conducted using Genstat v. 17 software (VSN International Ltd.).

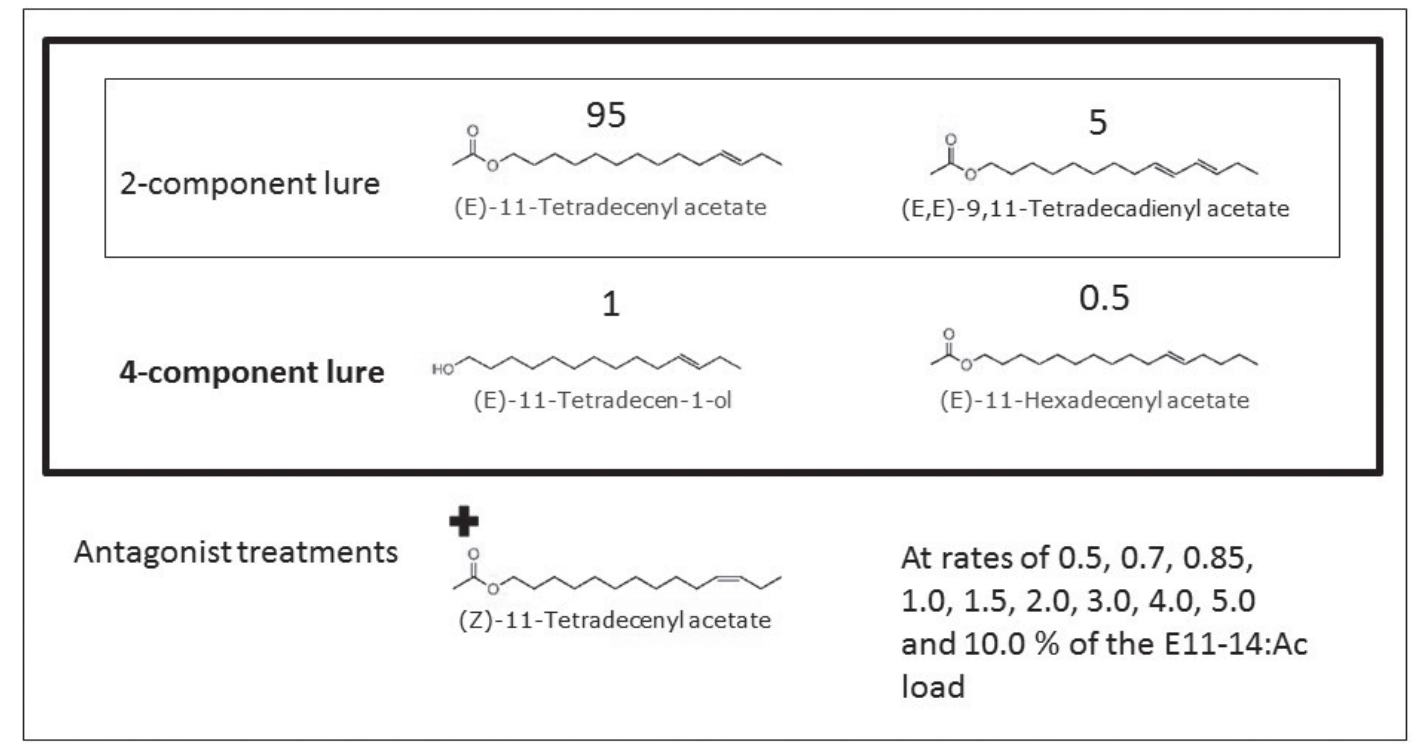

Figure 1 Structure of the compounds in the 2- and 4-component lures, and the added behavioural antagonist, Z11-14:Ac. 


\section{RESULTS}

The results of the full GLM analysis using all the tested variables indicated that there were no significant differences in the number of moths trapped between the blocked replicates and so this variable was dropped from the model. There was a significant decrease in the number of male E. postvittana caught in all pheromonebaited traps as the ratio of E11-14:Ac in the blend decreased $\left(\chi^{2}=108.74 ;\right.$ d.f. $\left.=9 ; \quad \mathrm{P}<0.001\right)$. Moth catch in the traps baited with the lure with the highest E11-14:Ac : Z11-14:Ac ratio (99.5:0.5) was predicted to be 21 times greater $(1 / 0.046)$ than catch in traps baited with the lowest purity lure (90:10) and was greater than all E11-14:Ac : Z11-14:Ac ratios except 99.3:0.7 (Table 1; Fig. 2). Traps baited with the four-component lures caught significantly more moths (1.57 times) than traps baited with the two-component lures ( $\chi 2=9.81 ;$ d.f. $=1 ; \mathrm{P}=0.002)$ (Table 1: Fig. 2 ).

Pair-wise comparisons of the catch of the two- or four-component lures within each E11-14:Ac: Z11-14:Ac ratio observed differences at the 99:1 $(\mathrm{P}=0.031)$ and $98: 2$ ratio $(\mathrm{P}=0.047)$. There were no observed differences in catch to the twoversus four-component lures at the other E1114:Ac : Z11-14:Ac ratios (Fig. 2).

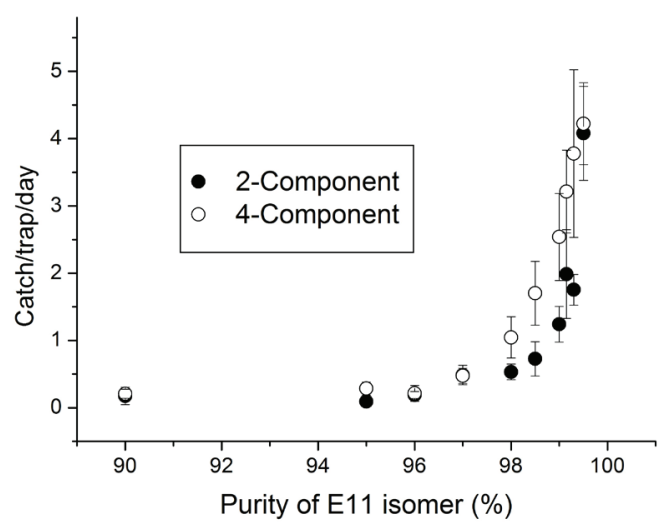

Figure 2 Mean catch per trap per day of Epiphyas postvittana with traps baited with two- or fourcomponent pheromones, and Z11-14:Ac added at different ratios to the main component, E1114:Ac. Bars show one standard error.

Table 1 Generalised Linear Model analysis of the effect of the ratio of E11-14:Ac: Z11-14:Ac in the blend and the number of pheromone components on catch of male E. postvittana. The results are compared to their relative reference factor (ratio of 99.5:0.5 or two components). The factor is the ratio of E1114:Ac : Z11-14:Ac. The estimate is the moth count of each ratio treatment relative to the 99.5:0.5 lure. The antilog of the estimate can be interpreted as the multiple by which the test factor is different to the reference factor.

\begin{tabular}{llcccc}
\hline Factor & Estimate & S.E. & $\mathrm{t}$ & $\mathrm{P}$ & Antilog of estimate \\
\hline 99.3:0.7 & -0.405 & 0.207 & -1.95 & 0.051 & 0.667 \\
99.15:0.85 & -0.467 & 0.211 & -2.21 & 0.027 & 0.627 \\
$99: 1$ & -0.787 & 0.235 & -3.35 & $<.001$ & 0.455 \\
$98.5: 1.5$ & -1.229 & 0.276 & -4.46 & $<.001$ & 0.293 \\
$98: 2$ & -1.660 & 0.328 & -5.06 & $<.001$ & 0.190 \\
$97: 3$ & -2.156 & 0.407 & -5.29 & $<.001$ & 0.116 \\
$96: 4$ & -3.026 & 0.607 & -4.99 & $<.001$ & 0.048 \\
$95: 5$ & -3.093 & 0.627 & -4.94 & $<.001$ & 0.045 \\
$90: 10$ & -3.075 & 0.621 & -4.95 & $<.001$ & 0.046 \\
Four components & 0.451 & 0.144 & .13 & 0.002 & 1.570 \\
\hline
\end{tabular}




\section{DISCUSSION}

The addition of the behavioural antagonist Z11-14:Ac in increasing proportions to twocomponent pheromone lures reduced the catch of male E. postvittana as found previously by Rumbo et al. (1993) and Stephens et al. (2008). Also, the present study showed high sensitivity to very small increases in Z11-14:Ac for the first time. Rumbo et al. (1993) showed Z11-14:Ac to be an attraction antagonist for E. postvittana when it was released within a wind tunnel from the same source as the pheromone at $10 \%$ of the pheromone level, but found no effect at $1 \%$. The results of the current study showed that even trace amounts of the Z11-14:Ac $(<0.5 \%$ of $\mathrm{E}$ isomer) were behaviourally active, as indicated by a significant reduction in catch from $99.5 \%$ to 99.15\% E11:14Ac purity.

Rumbo et al. (1993) observed some wind tunnel flight of male E. postvittana occurring when Z11-14:Ac was released at a concentration of $10 \%$. It is probable that more diluted openfield plumes experienced by the insects in the current trial as compared to doses experienced by the moths in a closed wind-tunnel system could explain the differences in where the threshold for antagonism occurs, although other cues such as host plant volatiles were also missing in the wind tunnel studies. The $10 \%$ concentration in the wind tunnel may have been overwhelming for the moths to respond to and lower levels in the field are likely to be more easily interpreted by them.

Addition of Z11-14:Ac in increasing proportions to the four-component lures also saw a reduction in catch, which is a new finding for this species. Overall, the four-component lures caught 1.6 times the number of moths caught in two-component lure traps. However, a significant difference in trap catch between the 2and 4-component lures was found only at ratios of 99:1 and 98:2 E11-14:Ac: Z11-14:Ac. This may be due to the sample size and large variation in trap catches within a treatment.

The results of this study clearly show that Z11$14: A c$ is a powerful antagonist to attraction in this species, even at very low levels. Conversely, a
1.6 fold improvement in catch (less antagonism) occurred with the incorporation of the two minor compounds ((E)-11-tetradecen-1-ol and (E)-11-hexadecenyl acetate) when Z1114: Ac was present, suggesting an interaction between the minor components and Z11-14:Ac. The specificity of olfactory receptor neurons (ORNs) allows insects to distinguish between compounds sharing very similar chemical structures, including pheromone compounds with different geometry and/or isomeric position of the double bond, as is the case with E11-14:Ac and Z11-14:Ac. In addition to these ORNs, some others are tuned not only to the conspecific sex pheromones, but those of closely related species (Zhang et al. 2015). It is probable that in its country of origin, Australia (Horak et al. 1988), where there are many congeneric species, there has been selection pressure on $E$. postvittana for specificity of signalling to avoid unsuccessful pairings with such moths. Epiphyas ashworthana, for example, reportedly uses the Z11 isomer as a sex pheromone (Whittle and Bellas, unpublished). The strong response to the antagonist, and interactions with other compounds incorporated in lures reported here and in previous publications, indicates that these compounds may be of critical importance to their effectiveness. It appears possible that current commercial two-component E. postvittana sex pheromone lures could be improved by consideration of how much Z11-14:Ac and (E)11-tetradecen-1-ol and (E)-11-hexadecenyl acetate are incorporated into them. Increases to attractiveness of lures for this species by addition of the minor compounds would require calibration for integrated pest management or other thresholds.

\section{ACKNOWLEDGEMENTS}

This work was funded as SSIF or Core Pipfruit sector research by the Ministry of Business Innovation and Employment. 


\section{REFERENCES}

Bellas TE, Bartell RJ, Hill A 1983. Identification of two components of the sex-pheromone of the moth, Epiphyas postvittana (Lepidoptera, Tortricidae). Journal of Chemical Ecology 9: 503-512.

Cossé AA, Todd JL, Baker TC 1998. Neurons discovered in male Helicoverpa zea antennae that correlate with pheromone-mediated attraction and interspecific antagonism. Journal of Comparative Physiology A 182: 585-594.

El-Sayed AM, Mitchell VJ, Manning LAM, Suckling DM 2011. New sex pheromone blend for the lightbrown apple moth, Epiphyas postvittana. Journal of Chemical Ecology 37: 640-646.

Horak M, Whittle CP, Bellas TE, Rumbo ER 1988. Pheromone gland components of some Australian tortricids in relation to their taxonomy. Journal of Chemical Ecology 14: 1163-1175.

Juárez ML, Ruiz MJ, Fernández PC, Goane L, Villagrán ME, Arce OEA, Armiñana A, Páez Jerez PG, de la Vega MH, Vera MT, Groot AT 2016. Communication interference in sympatrically occurring moth species. Entomologia Experimentalis et Applicata 158: 25-33.

Martin N, Moore K, Musto CJ, Linn CE 2016. Flight tunnel response of male European corn borer moths to cross-specific mixtures of European and Asian corn borer sex pheromones: evidence supporting a critical stage in evolution of a new communication system. Journal of Chemical Ecology 42: 5154.

Mo J, Glover M, Munro S, Beattie GAC 2006. Evaluation of mating disruption for control of lightbrown apple moth (Lepidoptera: Tortricidae) in citrus. Journal of Economic Entomology 99: 421-426.

Renou M, Guerrero A 2000. Insect parapheromones in olfaction research and semiochemical-based pest control strategies. Annual Review of Entomology 45: 605-630.
Rumbo E, Deacon S, Regan L 1993. Spatial discrimination between sources of pheromone and an inhibitor by the light-brown apple moth Epiphyas postvittana (Walker) (Lepidoptera: Tortricidae). Journal of Chemical Ecology 19: 953-962.

Stephens AEA, Suckling DM, El-Sayed AM 2008. Odour quality discrimination for behavioural antagonist compounds in three tortricid species. Entomologia Experimentalis et Applicata 127: 176-183.

Suckling DM, Shaw PW 1995. Large-scale trials of mating disruption of lightbrown apple moth in Nelson, New Zealand. New Zealand Journal of Crop and Horticultural Science 23: 127-137.

Suckling DM, Shaw PW, Khoo JGI, Cruickshank V 1990. Resistance management of lightbrown apple moth, Epiphyas postvittana (Lepidoptera: Tortricidae) by mating disruption. New Zealand Journal of Crop and Horticultural Science 18: 89-98.

Suckling DM, Sullivan TES, Stringer LD, Butler RC, Campbell DM, Twidle AM, Allen WJ, Mafra-Neto A, El-Sayed AM 2012. Communication disruption of light brown apple moth (Epiphyas postvittana) using a four-component sex pheromone blend. Crop Protection 42: 327-333.

Wu H, Xu M, Hou C, Huang LQ, Dong JF, Wang CZ 2015. Specific olfactory neurons and glomeruli are associated to differences in behavioral responses to pheromone components between two Helicoverpa species. Frontiers in Behavioral Neuroscience 9.

Zhang D, Löfstedt C 2015. Moth pheromone receptors: gene sequences, function, and evolution. Frontiers in Ecology and Evolution 3. 